\title{
Subcutaneous Trastuzumab Combined with Pertuzumab and Docetaxel as First-line Treatment of Advanced HER2-positive Breast Cancer
}

\author{
DIMITRA STEFANOU, STEFANIA KOKKALI, ELLI-SOPHIA TRIPODAKI, MARIA DRIZOU, \\ ELPIDA MAGOU, DIMOSTHENIS ZYLIS, MARIA PREVEZANOU, MATTHAIOS KAPIRIS, \\ DESPOINA NASI, ANNA NTOKOU, MARY DEDE and ALEXANDROS ARDAVANIS
}

First Medical Oncology Clinic, Saint Savvas Anticancer Hospital, Athens, Greece

\begin{abstract}
Background/Aim: Subcutaneous (s.c.) trastuzumab was introduced in the (neo)adjuvant setting, based on the non-inferiority results and patient preference. In the advanced setting, preliminary safety data have only been reported. We conducted an observational study of s.c. trastuzumab in combination with i.v. pertuzumab and docetaxel in the firstline setting of human epidermal growth factor receptor 2 (HER2)-positive advanced breast cancer. Patients and Methods: In this single-institution study, patients received 600 mg s.c. trastuzumab in combination with $840 \mathrm{mg}$ pertuzumab for the first cycle and $420 \mathrm{mg}$ for the following cycles, and $75-100 \mathrm{mg} / \mathrm{m}^{2}$ docetaxel, followed by maintenance with s.c. trastuzumab and pertuzumab until disease progression or unacceptable toxicity. Endpoints were efficacy and safety. Results: Forty patients were enrolled. The median number of cycles with docetaxel was six, while the median number of maintenance cycles was 21. With a median follow-up of 37 months, median progression-free survival and overall survival were 24 and 35 months. Conclusion: Subcutaneous trastuzumab in combination with pertuzumab and docetaxel is well tolerated and effective in HER2-positive advanced breast cancer.
\end{abstract}

Breast cancer is the most common cancer and the second leading cause of cancer-related death among women worldwide. It is recognized as a heterogeneous disease. Human epidermal growth factor receptor 2 (HER2) overexpression in breast cancer is related to a more

Correspondence to: Stefania Kokkali, First Medical Oncology Clinic, Saint Savvas Anticancer Hospital, Alexandras Avenue 171, 11522 Athens, Greece. Tel: +30 2106409227, Fax: +30 2106409483, e-mail: stefaniakokkali@yahoo.com

Key Words: Metastatic breast cancer, HER2-positive, subcutaneous trastuzumab. aggressive subtype. The addition of pertuzumab to trastuzumab and docetaxel significantly improved the median overall survival (OS) for patients with HER2-positive metastatic breast cancer (MBC). Thus, the combination of intravenous (i.v.) trastuzumab, pertuzumab and docetaxel is considered to be the standard first-line treatment in this setting $(1,2)$. Subcutaneous (s.c.) trastuzumab was first introduced in the (neo)adjuvant setting based on the results of the HannaH study reporting comparable safety and noninferiority $(3,4)$, as well as the PrefHer study pointing out patient preference for s.c. trastuzumab versus i.v. administration (5). This transition led to time and cost reductions (6). Based on that, we adopted a combination of s.c. trastuzumab, pertuzumab and docetaxel in patients with HER2-positive MBC. We present the results of our observational study.

\section{Patients and Methods}

Study design. This study was a retrospective single-arm analysis of fixed-dose s.c. trastuzumab combined with pertuzumab and docetaxel as first-line treatment in HER2-positive advanced breast cancer (metastatic or locally advanced). The data collected derived from a single center, the Saint-Savvas Anticancer Hospital, Athens, Greece. The primary endpoint of the study was to assess the efficacy of the combination by means of progression-free survival (PFS). Secondary endpoints were: i) Safety profile as determined by National Cancer Institute-Common Terminology Criteria for Adverse Events (NCI-CTCAE) v. 4.03 (7) and ii) overall survival (OS). All patients gave their written informed consent before undergoing study-specific procedures. The study design and conduct, as well as the informed consent document were approved by the Ethics committee/Institutional Review Board of Saint-Savvas Anticancer Hospital (163/6-7-2015).

Eligibility criteria. The main inclusion criterion was histological confirmation of locally advanced or recurrent or metastatic HER2positive breast cancer, treated with s.c. trastuzumab/pertuzumab/ docetaxel in the first-line setting at our center. Additional eligibility criteria included: age $>18$ years; Eastern Cooperative Oncology 
Group (ECOG) performance status (PS) of $0-2$ and adequate hematological, hepatic and renal function (hemoglobin $>9.5 \mathrm{~g} / \mathrm{dl}$; absolute neutrophil count $>1,500 / \mathrm{dl}$; total bilirubin $<1.5$ times the upper normal limit; and serum creatinine $<2.0 \mathrm{mg} / \mathrm{dl}$ ); left ventricular ejection fraction of $50 \%$ or more at baseline; measurable disease according to RECIST criteria 1.1 [complete response was defined as the disappearance of all target lesions; partial response was a $\geq 30 \%$ decrease in the sum of the largest diameter (SLD) of target lesions compared with baseline; progressive disease was a $\geq 20 \%$ increase in the SLD of target lesions compared with the smallest recorded SLD; stable disease was defined as either not sufficient shrinkage to qualify for complete or partial response, or not sufficient increase to qualify as progressive disease] (8).

Study treatment. The treatment schedule consisted of $600 \mathrm{mg}$ s.c. trastuzumab, $75-100 \mathrm{mg} / \mathrm{m}^{2}$ i.v. docetaxel and $840 \mathrm{mg}$ i.v. pertuzumab at the first cycle, followed by $420 \mathrm{mg}$ at subsequent cycles. The drugs were administered every 3 weeks. At least six cycles of chemotherapy were recommended; interruption and dose modifications were implemented according to standard guidelines. When chemotherapy was completed or discontinued due to toxicity or clinical judgment, pertuzumab and s.c. trastuzumab was continued as maintenance therapy until disease progression or unacceptable toxicity.

Evaluation of patients. Baseline evaluations included history, physical examination, hematological and biochemical tests and imaging studies, including computed tomographic (CT) scan of the chest and $\mathrm{CT}$ or magnetic resonance imaging (MRI) scan of the abdomen.

Physical examination, toxicity assessment, hematological and biochemical tests were performed before each cycle of treatment. Toxicity was graded according to the NCI CTCAE v4.03. Tumor response assessment (mainly by CT or MRI scans) was performed every three completed cycles of the study regimen, or earlier if clinically indicated, until documentation of progressive disease according to RECIST criteria 11 During the maintenance phase, imaging re-evaluation was performed every six cycles.

Statistical considerations. For survival analysis, the Kaplan-Meier method was used. PFS was defined as the time from the first cycle of study treatment to the first documented progression of disease or death. Patients without a disease-related event were censored at the last follow-up at which they were known to be progression-free. OS was defined as the time from the first cycle of the study treatment to death. Patients who were still alive were censored on the date of their last known follow-up visit.

\section{Results}

Patient characteristics and treatment exposure. Between April 2014 and December 2017, 40 women were treated. The median age at diagnosis was 62 years. All patients had ECOG PS 0-3. Patient characteristics are summarized in Table I.

The vast majority of patients had metastatic disease, while only four had locally advanced breast cancer. Forty percent of our patients had visceral metastases. The most common sites of metastatic disease included the lungs, liver, lymph nodes and bones. A little less than half of patients had hormonal
Table I. Patient baseline demographics and disease characteristics.

\begin{tabular}{|c|c|}
\hline Parameter & Value \\
\hline \multicolumn{2}{|l|}{ Age } \\
\hline Median (range), years & $62(37-85)$ \\
\hline$>65$ Years, $\mathrm{N}(\%)$ & $16(40)$ \\
\hline$\leq 65$ Years, $\mathrm{N}(\%)$ & $24(60)$ \\
\hline \multicolumn{2}{|l|}{ HR status, $\mathrm{N}(\%)^{*}$} \\
\hline $\mathrm{ER}^{+}$or $\mathrm{PR}^{+}$or both & $19(47.5)$ \\
\hline $\mathrm{ER}^{-}$and $\mathrm{PR}^{-}$ & $21(52.5)$ \\
\hline \multicolumn{2}{|l|}{ ECOG PS, N (\%) } \\
\hline 0 or 1 & $28(70 \%)$ \\
\hline 2 or 3 & $12(30)$ \\
\hline \multicolumn{2}{|c|}{ Disease presentation at inclusion, $\mathrm{N}(\%)$} \\
\hline De novo & $13(32.5)$ \\
\hline Relapse & $27(67.5)$ \\
\hline Visceral metastases, $\mathrm{N}(\%)$ & $16(40)$ \\
\hline \multicolumn{2}{|c|}{ (Neo)adjuvant treatment in patients with relapse, $\mathrm{N}$} \\
\hline Anthracyclines & 18 \\
\hline Taxanes & 17 \\
\hline Trastuzumab & 15 \\
\hline \multicolumn{2}{|c|}{ Time from last trastuzumab for early $\mathrm{BC}(\mathrm{N}=15)$} \\
\hline Median (range), months & $34(10-144)$ \\
\hline
\end{tabular}

HR: Hormone receptor status, ER: estrogen receptor, PR: progesterone receptor, ECOG: Eastern Cooperative Oncology Group, PS: performance status, BC: breast cancer. *Refers to primary breast tumor in 37 patients; in the remaining three patients with no breast biopsy, it refers to biopsy of metastasis.

receptor (HR)-positive disease. Approximately one-third of them had de novo MBC, while the remaining had been treated with adjuvant or neoadjuvant chemotherapy with or without trastuzumab. Eleven patients $(40.7 \%)$ of the latter group underwent biopsy from a metastatic site. All patients received s.c. trastuzumab in combination with pertuzumab and docetaxel. The median number of cycles of chemotherapy per patient was 6 (range 4-12), while the median number of cycles of trastuzumab-pertuzumab was 21 (0-65). HR-positive patients received hormonal treatment during the maintenance phase. Dose modification of docetaxel was required in a minority of patients due to hematological or other toxicity.

Efficacy. Data were analyzed on September 4, 2018. With a median follow-up period of 37 months, 31 patients were alive. PFS was between 14.4 and 33.6 months, with a median value of 24 months, as shown in Figure 1. Sixteen patients were still on treatment, 23 patients experienced disease progression and two patients had been lost to follow-up. Seven patients had died, six of them due to disease progression. OS was between 27.9 and 42.1 months, with a median value of 35 months (Figure 2).

Safety. The most common adverse events included neutropenia, peripheral neuropathy and diarrhea, related 


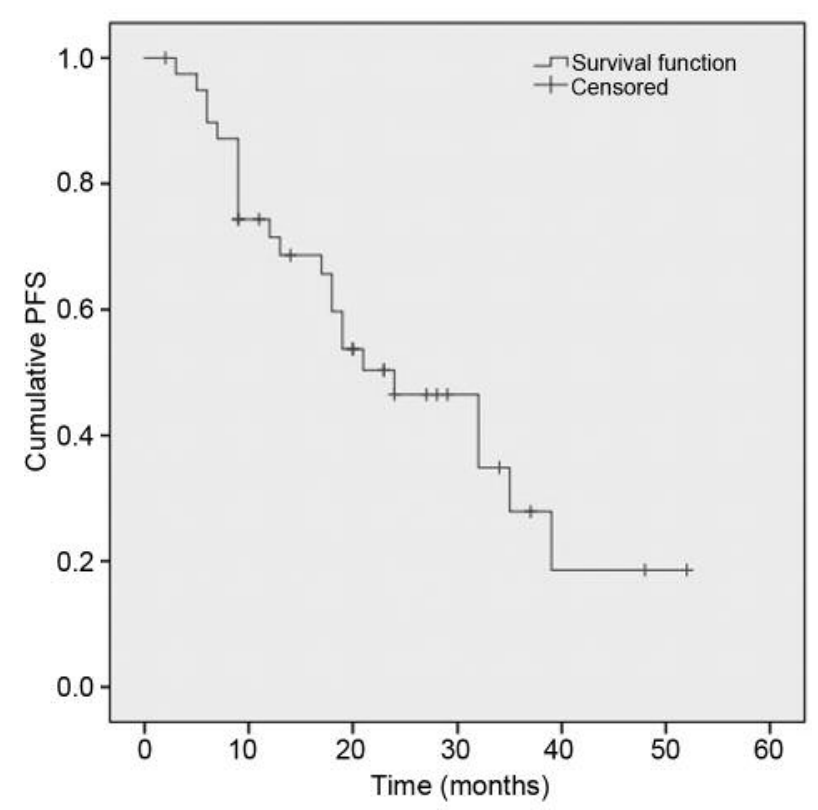

Figure 1. Kaplan-Meier curve of progression-free survival (PFS). The whole cohort of patients was included in the analysis.

mainly to docetaxel. Four patients needed hospitalization due to toxicity. One toxic death was observed due to diarrhea and acute renal failure. Cardiac toxicities related to trastuzumabpertuzumab occurred in four patients and led to permanent discontinuation of therapy in one patient. No other toxicity related to s.c. trastuzumab was noted, notably no local injection-site reaction.

\section{Discussion}

The advent of anti-HER2 targeted therapy has turned HER2positive MBC into a chronic disease. Trastuzumab has a major impact on the treatment of HER2-positive MBC, especially in combination with chemotherapy (9). The CLEOPATRA trial demonstrated that the addition of pertuzumab to trastuzumab plus chemotherapy improved survival, with a median OS of 56.5 months $(1,2,10)$. Moreover, s.c. delivery of trastuzumab represents an alternative to the i.v. route and is associated with various practical advantages $(5,6,11)$. In the adjuvant/neoadjuvant setting, the HannaH study reported for the first-time similar event-free survival with s.c. and i.v. Trastuzumab $(3,12)$, whereas pharmacokinetics analysis demonstrated that the fixed $600 \mathrm{mg}$ s.c. dose of trastuzumab exposes the patients to the drug concentration required for efficacy (13). In addition, the PrefHer study revealed that the vast majority of both patients and healthcare professionals opted for s.c.

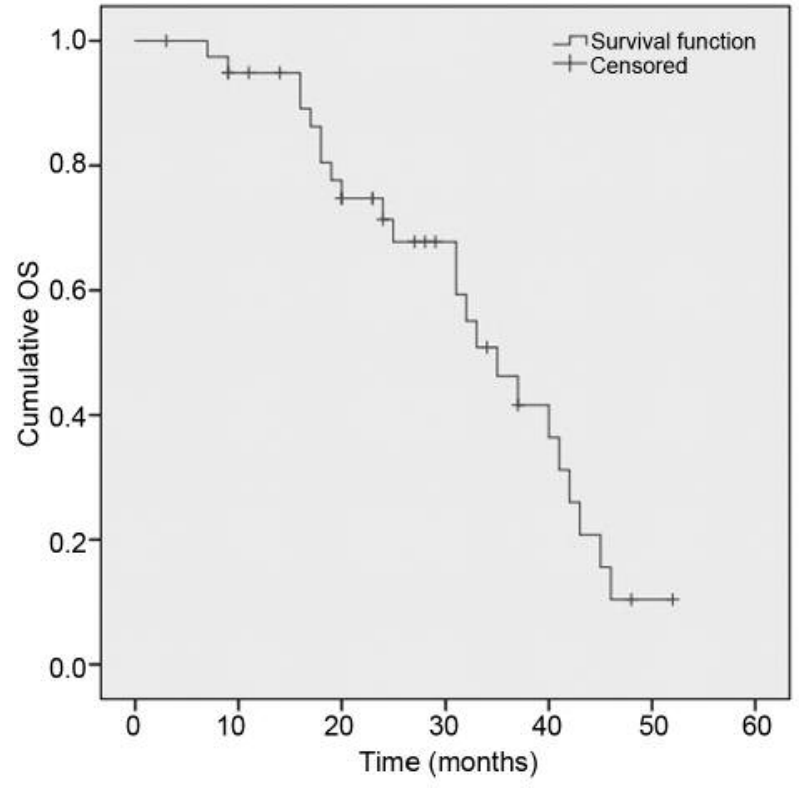

Figure 2. Kaplan-Meier curve of overall survival (OS). The whole cohort of patients was included in the analysis.

administration (5). A reduction of almost 1 minute in chair time per patient and 15 minutes in healthcare professional active time was shown by time-consumption analysis (14).

Our single-center observational study, which was initiated by the above positive findings in early breast cancer, investigated the combination of pertuzumab with s.c. trastuzumab and docetaxel in HER2-positive MBC. Subpopulation analysis was not carried out due to the small number of patients. Preliminary results of two phase IIIb multicenter studies of s.c. trastuzumab in the metastatic setting were presented recently at the San Antonio Breast Cancer Symposium. The MetaPHER trial investigated the safety profile of s.c. trastuzumab in combination with pertuzumab and docetaxel, which was as expected from the CLEOPATRA trial (15). Similar results were obtained from the combination of s.c. trastuzumab with pertuzumab and any taxane in the SAPPHIRE study (16). Efficacy data of both trials are still awaited.

In our study, the median PFS was 24 months, which was longer compared to the pivotal trial results (2). In contrast, median OS in our study was significantly shorter than that in CLEOPATRA. It should be noted that there are some differences between the population of our study and that of the CLEOPATRA trial. In our study, fewer patients had visceral metastasis (40\% vs. $78 \%$ ). On the other hand, the population of our study had a higher rate of previous adjuvant/neoadjuvant trastuzumab (37.5\% vs. 10\%), leading 
to less clinical benefit of Trastuzumab (17). These differences, as well as the much lower number of patients in our study, do not allow a reliable comparison. In addition, real-world studies in general differ from randomized controlled trials since the study drug is evaluated in a broader population, similarly to the real clinical setting, leading to a more thorough drug benefit-risk ratio evaluation. A real-world USA retrospective study of the same drug combination reported a similar median PFS of 16.9 months (18), whereas an exceptionally high PFS of 27.8 months was reported by an Italian real-world study (19), vs. 18.7 months in the CLEOPATRA trial.

Regarding adverse events, the observed safety profile, including cardiac toxicity, is in accordance with what has been previously reported in the CLEOPATRA, PERUSE and MetaPHER trials $(15,20,21)$, indicating that the combination s.c. trastuzumab with pertuzumab and docetaxel is safe and well tolerated.

It should be noted that in $40.7 \%$ of our patients with recurrent breast cancer, a biopsy from a metastatic site had been performed. Specifically, discordance in HR status between the primary tumor and the corresponding relapse was observed in three out of 11 patients who underwent biopsy. In contrast, changes were rare as far as HER2 status was concerned.

In the case of disease progression, the majority of patients received trastuzumab-emtansine as second-line treatment (11/23 patients). The remaining patients received eribulin with or without trastuzumab, trastuzumab plus letrozol or lapatinib, and other chemotherapy regimens not containing anti-HER2 agents (gemcitabine/vinorelbine, paclitaxel/bevacizumab, nabpaclitaxel). Five patients among the 23 with progressive disease experienced progression only in the central nervous system. They received whole-brain radiotherapy due to multiple brain metastases. Concerning systemic treatment, two of them received trastuzumab/ pertuzumab/capecitabine, one trastuzumab-emtansine, one capecitabine-lapatinib and one paclitaxel/bevacizumab. Despite the fact that the CLEOPATRA regimen was found to delay the onset of central nervous system metastasis (22), we consider these patients a special subgroup, not necessarily requiring systemic treatment modification, provided they receive local treatment.

Our study has strengths and limitations. On the one hand, we evaluated the combination of s.c. trastuzumab with pertuzumab and docetaxel in the metastatic setting and tried to fill the knowledge gap between the existing evidence for s.c. trastuzumab in the (neo)adjuvant setting and its use in HER2-positive advanced breast cancer. In addition, this evaluation was performed in a real-world setting, providing important information about actual clinical practice. On the other hand, the number of patients included in our study was small, as they were treated at a single-center. The inherent limitations of retrospective analysis are also to be noted.
In summary, the combination of s.c. trastuzumab with pertuzumab and docetaxel is well tolerated and effective in patients with HER2-positive MBC.

\section{References}

1 Baselga J, Cortés J, Kim S-B, Im SA, Hegg R, Im YH, Roman L, Pedrini JL, Pienkowski T, Knott A, Clark E, Benyunes MC, Ross G and Swain SM: Pertuzumab plus trastuzumab plus docetaxel for metastatic breast cancer. N Engl J Med 366: 109119, 2012.

2 Swain SM, Kim S-B, Cortés J, Ro J, Semiglazov V, Campone M, Ciruelos E, Ferrero JM, Schneeweiss A, Knott A, Clark E, Ross G, Benyunes MC and Baselga J: Pertuzumab, trastuzumab, and docetaxel for HER2-positive metastatic breast cancer (CLEOPATRA study): overall survival results from a randomised, double-blind, placebo-controlled, phase 3 study. Lancet Oncol 14: 461-471, 2013.

3 Jackisch C, Hegg R, Stroyakovskiy D, Ahn JS, Melichar B, Chen SC, Kim SB, Lichinitser M, Starosławska E, Kunz G, Falcon S, Chen ST, Crepelle-Fléchais A, Heinzmann D, Shing $\mathrm{M}$ and Pivot X: HannaH phase III randomised study: Association of total pathological complete response with event-free survival in HER2-positive early breast cancer treated with neoadjuvantadjuvant trastuzumab after 2 years of treatment-free follow-up. Eur J Cancer 62: 62-75, 2016.

4 Jackisch C, Kim S-B, Semiglazov V, Melichar B, Pivot X, Hillenbach C, Stroyakovskiy D, Lum BL, Elliott R, Weber HA and Ismael G: Subcutaneous versus intravenous formulation of trastuzumab for HER2-positive early breast cancer: updated results from the phase III HannaH study. Ann Oncol 26: 320325, 2015.

5 Pivot X, Verma S, Fallowfield L, Müller V, Lichinitser M, Jenkins V, Sánchez Muñoz A, Machackova Z, Osborne S, Gligorov J; PrefHer Study Groupe: Efficacy and safety of subcutaneous trastuzumab and intravenous trastuzumab as part of adjuvant therapy for HER2-positive early breast cancer: Final analysis of the randomised, two-cohort PrefHer study. Eur J Cancer 86: 82-90, 2017.

6 Tjalma WAA, Van den Mooter T, Mertens T, Bastiaens V, Huizing MT and Papadimitriou K: Subcutaneous trastuzumab (Herceptin) versus intravenous trastuzumab for the treatment of patients with HER2-positive breast cancer: A time, motion and cost assessment study in a lean operating day care oncology unit. Eur J Obstet Gynecol Reprod Biol 221: 46-51, 2018.

7 National Cancer Institute-Common Terminology Criteria for Adverse Events (NCI-CTCAE) v. 4.03. https://ctep.cancer.gov/ protocolDevelopment/electronic_applications/docs/CTCAE_v5_ Quick_Reference_8.5x11.pdf

8 Eisenhauer EA, Therasse P, Bogaerts J, Schwartz LH, Sargent D, Ford R, Dancey J, Arbuck S, Gwyther S, Mooney M, Rubinstein L, Shankar L, Dodd L, Kaplan R, Lacombe D and Verweij J: New response evaluation criteria in solid tumours: Revised RECIST guideline (version 1.1). Eur J Cancer 45: 228-247, 2009.

9 Debiasi M, Reinert T, Kaliks R, Amorim G, Caleffi M, Sampaio $\mathrm{C}$, Fernandes GDS and Barrios $\mathrm{CH}$ : Estimation of premature deaths from lack of access to anti-HER2 therapy for advanced breast cancer in the Brazilian Public Health System. J Glob Oncol 3: 201-207, 2017. 
10 Swain SM, Baselga J, Kim S-B, Ro J, Semiglazov V, Campone M, Ciruelos E, Ferrero JM, Schneeweiss A, Heeson S, Clark E, Ross G, Benyunes MC, Cortés J: Pertuzumab, trastuzumab, and docetaxel in HER2-positive metastatic breast cancer. N Engl J Med 372: 724-734, 2015.

11 Pivot X, Spano JP, Espie M, Cottu P, Jouannaud C, Pottier V, Moreau L, Extra JM, Lortholary A, Rivera P, Spaeth D, AttarRabia H, Benkanoun C, Dima-Martinez L, Esposito N and Gligorov J: Patients' preference of trastuzumab administration (subcutaneous versus intravenous) in HER2-positive metastatic breast cancer: Results of the randomised MetaspHer study. Eur J Cancer 82: 230-236, 2017.

12 Ismael G, Hegg R, Muehlbauer S, Heinzmann D, Lum B, Kim SB, Pienkowski T, Lichinitser M, Semiglazov V, Melichar B and Jackisch C: Subcutaneous versus intravenous administration of (neo)adjuvant trastuzumab in patients with HER2-positive, clinical stage I-III breast cancer (HannaH study): A phase 3 , open-label, multicentre, randomised trial. Lancet Oncol 13: 869878, 2012.

13 Quartino AL, Hillenbach C, Li J, Li H, Wada RD, Visich J, Li C, Heinzmann D, Jin JY and Lum BL: Population pharmacokinetic and exposure-response analysis for trastuzumab administered using a subcutaneous "manual syringe" injection or intravenously in women with HER2-positive early breast cancer. Cancer Chemother Pharmacol 77: 77-88, 2016.

14 De Cock E, Pivot X, Hauser N, Verma S, Kritikou P, Millar D and Knoop A: A time and motion study of subcutaneous versus intravenous trastuzumab in patients with HER2-positive early breast cancer. Cancer Med 5: 389-397, 2016.

15 Kümmel S, Abraham J, Martín M, Crepelle-Fléchais A, Swat A, Nüesch E, Shing $M$ and Tondini C: MetaPHER phase IIIb multicenter, open-label, single-arm safety study of subcutaneous trastuzumab in combination with pertuzumab and docetaxel in patients with HER2-positive advanced breast cancer: First results. Cancer Res 77(4 Suppl): abstr P4-21-42, 2017.

16 Woodward N, De Boer R, Redfern A, White M, Young J, Truman $\mathrm{M}$ and Beith J: Interim results from the first open-label, multicentre, phase IIIb study investigating the combination of pertuzumab with subcutaneous trastuzumab and a taxane in patients with HER2-positive metastatic breast cancer (SAPPHIRE). Cancer Res 76(4 Suppl): abstr P4-14-12, 2016.
17 Rier HN, Levin MD, van Rosmalen J, Bos MMEM, Drooger JC, de Jong P, Portielje JEA, Elsten EMP, Ten Tije AJ, Sleijfer S and Jager A: First-line palliative HER2-targeted therapy in HER2positive metastatic breast cancer is less effective after previous adjuvant trastuzumab-based therapy. Oncologist 22: 901-909, 2017.

18 Robert NJ, Goertz H-P, Chopra P, Jiao X, Yoo B, Patt D and Antao V: HER2-positive metastatic breast cancer patients receiving pertuzumab in a community oncology practice setting: treatment patterns and outcomes. Drugs Real World Outcomes 4: 1-7, 2017.

19 De Placido S, Giuliano M, Schettini F, Von Arx C, Buono G, Riccardi F, Cianniello D, Caputo R, Puglisi F, Bonotto M, Fabi A, Bilancia D, Ciccarese M, Lorusso V, Michelotti A, Bruzzese D, Veneziani BM, Locci M, De Laurentiis M and Arpino G: Human epidermal growth factor receptor 2 dual blockade with trastuzumab and pertuzumab in real life: Italian clinical practice versus the CLEOPATRA trial results. Breast 38: 86-91, 2018.

20 Swain SM, Ewer MS, Cortés J, Amadori D, Miles D, Knott A, Clark E, Benyunes MC, Ross G and Baselga J: Cardiac tolerability of pertuzumab plus trastuzumab plus docetaxel in patients with HER2-positive metastatic breast cancer in CLEOPATRA: a randomized, double-blind, placebo-controlled phase III study. Oncologist 18: 257-264, 2013.

21 Bachelot T, Puglisi F, Ciruelos E, Peretz-Yablonski T, Schneeweiss A, Restuccia E, Easton V, Lindegger N and Miles D: Preliminary safety and efficacy of first-line pertuzumab combined with trastuzumab and taxane therapy for HER2positive locally recurrent/metastatic breast cancer (PERUSE). Cancer Res 77(4 Suppl): abstr P4-21-04, 2017.

22 Swain SM, Baselga J, Miles D, Im YH, Quah C, Lee LF, Cortés $\mathrm{J}$ : Incidence of central nervous system metastases in patients with HER2-positive metastatic breast cancer treated with pertuzumab, trastuzumab, and docetaxel: results from the randomized phase III study CLEOPATRA. Ann Oncol 25: 11161121,2014
Received October 1, 2018

Revised October 20, 2018

Accepted October 23, 2018 